\title{
Prognostic Stratification for IDH-wild-type Lower- grade Astrocytoma by Sanger Sequencing and Copy-number Variation Analysis with MLPA.
}

\section{Yasuhide Makino}

Kyoto University Graduate School of Medicine

Yoshiki Arakawa ( $\nabla$ yarakawa@kuhp.kyoto-u.ac.jp)

Kyoto University Graduate School of Medicine

\section{Ema Yoshioka}

Osaka National Hospital

\section{Tomoko Shofuda}

Osaka National Hospital

\section{Takeshi Kawauchi}

Kyoto University Graduate School of Medicine

\section{Yukinori Terada}

Kyoto University Graduate School of Medicine

\section{Masahiro Tanji}

Kyoto University Graduate School of Medicine

\section{Daisuke Kanematsu}

Osaka National Hospital

Yohei Mineharu

Kyoto University Graduate School of Medicine

\section{Susumu Miyamoto}

Kyoto University Graduate School of Medicine

\section{Yonehiro Kanemura}

Osaka National Hospital

\section{Research Article}

Keywords: astrocytoma, cIMPACT-NOW, MLPA (multiplex ligation-dependent amplification), IDH, TERTp, EGFR, PTEN

Posted Date: February 16th, 2021

DOI: https://doi.org/10.21203/rs.3.rs-198063/v1 
License: (c) (i) This work is licensed under a Creative Commons Attribution 4.0 International License. Read Full License

Version of Record: A version of this preprint was published at Scientific Reports on July 13th, 2021. See the published version at https://doi.org/10.1038/s41598-021-93937-8. 


\section{Abstract}

The characteristics of $I D H$-wild-type lower-grade astrocytoma remain unclear. According to cIMPACT-NOW update 3, IDH-wild-type astrocytomas with any of the following factors show poor prognosis: combination of chromosome 7 gain and 10 loss (+ 7/-10), and/or EGFR amplification, and/or TERT promoter (TERTp) mutation. Multiplex ligation-dependent probe amplification (MLPA) can detect copy number variations with a reasonable cost. The purpose of this study was to find precise and costeffective method for stratifying the prognosis of IDH-wild-type astrocytomas. Sanger sequencing, MLPA, and quantitative methylation-specific PCR were performed for 42 IDH-wild-type lower-grade astrocytomas surgically treated at Kyoto University Hospital, and overall survival was analyzed for 40 patients who underwent first surgery. Of the 42 IDH-wild-type astrocytomas, 21 were classified as grade 4 in cIMPACTNOW update 3 criteria and all of them had either TERTp mutation or EGFR amplification. Kaplan-Meier analysis confirmed the prognostic significance of cIMPACT-NOW criteria, and WHO grade was also prognostic. Cox regression hazard model identified PTEN loss and PDGFRA amplification as independent significant prognostic indicators (Risk ratio of 9.75, $p<0.001$ and 13.9, $p=0.002$ ). The classification recommended by cIMPACT-NOW update 3 could be completed using Sanger sequencing and MLPA. Survival analysis revealed PTEN and PDGFRA were significant prognostic factors for IDH-wild-type lowergrade astrocytoma.

\section{Introduction}

Glioma is a common tumour type originating in the human brain. ${ }^{1}$ Glioblastoma, grade IV (GBM) is the most aggressive and major subtype of glioma, while diffuse astrocytoma, grade II (DA) and anaplastic astrocytoma, grade III (AA) are lower-grade astrocytomas. All these pathological entities had been classified mainly based on histology in the 2007 WHO classification of central nervous system tumours. ${ }^{2} I D H$ mutation is widely recognised as a good predictor of survival among patients with glioma, ${ }^{3}$ and codeletion of chromosome $1 p$ and $19 q(1 p / 19 q$ codeletion) has been associated with oligodendroglioma and suggests longer survival. ${ }^{4}$ Based on such findings, the WHO 2016 classification combined genetic profiling with a grading system based on the WHO 2007 classification.

Oligodendroglioma was defined as a glioma with both $I D H$ mutation and $1 p / 19 q$ codeletion, and the $\mathrm{K} 27 \mathrm{M}$ mutation in $\mathrm{H} 3 \mathrm{~F} 3 \mathrm{~A}$ or less common $\mathrm{HIST} 1 \mathrm{H} 3 \mathrm{~B}$ was included in the criteria for diffuse midline glioma, H3K27M-mutant. ${ }^{5}$

It is well known that $I D H$-mutant and $I D H$-wild type astrocytomas shows distinct genetic profiles and prognosis. ${ }^{3,6-8}$ However, the disease-defining genetic alterations of IDH-wild type astrocytomas had not been revealed, ${ }^{5,9}$ and their diagnosis were remained to depend on the histological findings. On the other side, some studies concluded a substantial subset of IDH-wild-type lower-grade astrocytomas with poor survival course like as IDH-wild-type GBMs. ${ }^{7,9-14}$ Based on these studies, a previous report by the Consortium to Inform Molecular and Practical Approaches to CNS Tumour Taxonomy (cIMPACT-NOW) update 3 proposed that certain IDH-wild-type diffuse astrocytomas show a poor clinical course similar to 
IDH-wild-type glioblastomas, and that the characteristics of these tumours were a result of at least one of three genetic profiles: combined whole chromosome 7 gain and whole chromosome 10 loss (+7/-10); and/or EGFR amplification; and/or TERT promoter (TERTp) mutation. ${ }^{15}$ These three profiles have been recommended for inclusion in the next classification, with this specific type of astrocytoma to be diagnosed as "diffuse astrocytic glioma, IDH-wildtype, with molecular features of glioblastoma, WHO grade $I V$ ", referred to as "astrocytoma, grade 4" in the present study. Like these things, copy-number variation (CNV) as well as mutations have been to make clear classification of $I D H$-wild type astrocytomas corresponding to clinical prognosis.

In general, the hot spot mutations of $I D H 1 / 2$ and TERTp can be detected by Sanger sequencing $3,16-18$ and $1 p / 19 q$ codeletion can be examined by fluorescence in situ hybridisation or multiplex ligation-dependent probe amplification (MLPA). ${ }^{19,20}$ MLPA can also detect copy-number alterations in EGFR and PTEN, ${ }^{19,20}$ which are located on chromosome $7 p$ and $10 q$, respectively. However, whole-chromosomal alterations in chromosomes 7 and 10 were difficult to detect using only one MLPA kit, and are reportedly better ascertained by single nucleotide polymorphism array, ${ }^{11,14}$ DNA methylation array, ${ }^{9,13}$ array-based comparative genomic hybridisation, ${ }^{14,21}$ or next-generation sequencing. ${ }^{12}$ Unfortunately, those methods are difficult to conduct in many local institutes and hospitals, and thus are less than ideal as global standards. MLPA can target multiple different sequences in a single PCR experiment and can detect small and fragmented DNA. ${ }^{19,22}$ And all appliances needed to introduce the method is thermal cycler and capillary sequencer. MLPA has potential to detect poor prognosis subgroups in IDH-wild type astrocytomas like as "astrocytoma, grade 4", but the feasibility of this method is needed to be evaluated.

\section{Results}

Between August 19th, 2010 and December 19th, 2019, a total of 291 samples were resected from 257 patients in Kyoto University Hospital and cryopreserved. All samples received integrated diagnosis as described above, and 42 tumours from 42 patients ( 31 men, 11 women) were classified as IDH-wild-type astrocytoma. The characteristics of these 42 tumours are described in Table 1. The tumours comprised $18 \mathrm{IDH}$-wild-type DAs and $24 \mathrm{IDH}$-wild-type AAs, and median age at resection was 55.5 years (range, 5-85 years). No significant differences in age or sex were noted between tumour subtypes. Forty tumours were removed in first surgeries, comprising 18 DAs and $22 \mathrm{AAs}$, and no difference in age was evident between subtypes. However, initial treatments for AAs included chemoradiation therapy more frequently, whereas observation was selected more in DAs. The initial postsurgical treatments of these patients were 9 chemoradiation therapies and 1 chemotherapy for DAs, and 19 chemoradiation therapies and 1 chemotherapy for AAs, while observation with regular imaging without treatment was selected for 8 DAs and 2 AAs. 
Table 1

Characteristics of all patients included in the present study

\begin{tabular}{|c|c|c|c|c|}
\hline & Total & DA, IDHwt & AA, IDHwt & p-value* \\
\hline \multicolumn{5}{|l|}{ All tumours } \\
\hline Number, $\mathrm{n}$ & 42 & 18 & 24 & \\
\hline Sex, men, $\mathrm{n}$ & 31 & 13 & 18 & 1 \\
\hline Age, year, median (range) & $55.5(5-85)$ & $56.5(5-85)$ & $55(8-85)$ & 0.964 \\
\hline cIMPACT-NOW update3 "grade4", n & 21 & 9 & 12 & 1 \\
\hline TERTp mutation, $\mathrm{n}$ & 18 & 9 & 9 & 0.533 \\
\hline EGFR amplification, $\mathrm{n}$ & 8 & 1 & 7 & 0.109 \\
\hline EGFR gain and PTEN loss, $\mathrm{n}$ & 6 & 3 & 3 & 1 \\
\hline PTEN loss, $\mathrm{n}$ & 11 & 3 & 8 & 0.299 \\
\hline PDGFR amplification, $\mathrm{n}$ & 4 & 0 & 4 & 0.122 \\
\hline CDKN2A homozygous loss, $\mathrm{n}$ & 8 & 1 & 7 & 0.109 \\
\hline MGMTp hypermethylation, $\mathrm{n}$ & 13 & 6 & 7 & 1 \\
\hline \multicolumn{5}{|l|}{ Patients for survival analysis } \\
\hline Number, $\mathrm{n}$ & 40 & 18 & 22 & \\
\hline age, year, median (range) & $57(5-85)$ & $56.5(5-85)$ & $59(8-85)$ & 0.725 \\
\hline \multicolumn{5}{|l|}{ Initial treatment } \\
\hline Chemoradiation therapy, $\mathrm{n}$ & 28 & 9 & 19 & 0.0246 \\
\hline Chemotherapy, n & 2 & 1 & 1 & \\
\hline Observation, $\mathrm{n}$ & 10 & 8 & 2 & \\
\hline \multicolumn{5}{|c|}{$\begin{array}{l}\text { * The p-values between groups of DA and AA are calculated with Student's t-test for age and with } \\
\text { Fisher's exact test for the others. }\end{array}$} \\
\hline
\end{tabular}

The detailed status of mutations and alterations in the $42 \mathrm{IDH}$-wild-type astrocytomas are shown in Fig. 1-A. TERTp mutation was detected in 18 IDH-wild-type astrocytomas (42.9\%), comprising 9 DAs (50\%) and 9 AAs (37.5\%). Again, no significant difference was seen between these two tumour types. EGFR amplification was seen in 8 IDH-wild-type astrocytomas (19.0\%), comprising 1 DA (5.6\%) and 7 AAs $(29.2 \%)$, tending to be slightly more frequent in AAs than in DAs $(\mathrm{p}=0.109)$. The combination of $E G F R$ gain and PTEN loss (+ EGFR/-PTEN) was detected in 6 IDH-wild-type astrocytomas (14.3\%), comprising 3 
DAs (16.7\%) and 3 AAs (12.5\%), showing no significant difference. All DAs with EGFR amplification or + EGFR/-PTEN showed TERTp mutation, so all DAs diagnosed as "astrocytoma, grade 4" were equivalent to DAs with TERTp mutation, while 3 TERTp-wild type AAs showed EGFR amplification. Interestingly, no astrocytomas with + EGFR/-PTEN lacked the TERTp mutation.

\section{Classification of lower-grade astrocytomas, IDH-wild type}

The results of classification of IDH-wild-type lower-grade astrocytomas are shown in Fig. 1-B. TERTp mutation was detected in 18 of all $42 \mathrm{IDH}$-wild-type astrocytomas, and these 18 tumours were classified as "astrocytoma, grade 4" from Sanger sequence alone. In the 24 tumours without TERTp mutation, MLPA showed EGFR amplification in 3 AAs, and no tumours with + EGFR/-PTEN. As a result, 21 of all 42 tumours were classified as "astrocytoma, grade 4", comprising 9 DAs and 12 AAs. In our cohort, all instances of "astrocytoma, grade 4" were able to be diagnosed by the combination of Sanger sequence and MLPA.

\section{Correlations with tumour profiles}

Correlations between all pairs of the following factors were analysed: age at diagnosis, TERTp mutation, WHO grade, 06-methylguanine-DNA methyltransferase promoter (MGMTp) hyper methylation, and copy number alterations of EGFR, PTEN, CDKN2A, PDGFRA, MDM2, CDK4, NFKBIA, and TP53. TERTp mutation, EGFR amplification, CDKN2A homozygous loss, CDK4 gain or amplification (gain/amplification), CDK4 amplification, and MGMTp hypermethylation correlated with higher age at diagnosis $(p=0.0157, p=$ $0.0382, p=0.0272, p=0.0222$, and $p=0.0045$, respectively). Statistical correlations were detected between any two of TERTp mutation, EGFR gain/amplification and PTEN loss (TERTp and EGFR gain/amplification, odds ratio 5.91, $\mathrm{p}=0.0236$; TERTp mutation and PTEN loss, odds ratio $11, \mathrm{p}=0.004$; EGFR gain/amplification and PTEN loss, odds ratio 9.38, $\mathrm{p}=0.0304)$. Another correlation was seen between PDGFRA gain/amplification and CDKN2A homozygous loss (odds ratio, 7.78; $\mathrm{p}=0.0195$ ). WHO grade correlated significantly with MDM2 loss and MDM2 hemizygous loss $(p=0.0054$ and $p=0.014$, respectively). On the other hand, WHO grade showed no significance in the other profiles (Table 1).

\section{Survival analysis of lower-grade astrocytomas, IDH-wild type}

Clinical outcomes were calculated for the 40 cases in which the tumours were removed in first surgeries, including 18 DAs and 22 AAs. "Astrocytoma, grade 4" showed significantly shorter overall survival (OS) compared with other tumours in all astrocytomas $(\mathrm{p}=0.0149)$ and DAs $(\mathrm{p}=0.0036)$, but not in AAs $(\mathrm{p}=$ 0.1288) (Fig. 2-A). TERTp mutations were significantly associated with poor OS in all astrocytomas $(\mathrm{p}=$ $0.0228)$, and in DAs $(p=0.0036)$, but not in AAs $(p=0.0884)$ (Fig. $2-B)$. EGFR amplification was a significant factor for OS only in all astrocytomas $(p=0.0401)$, not in diffuse DAs $(p=0.7893)$ or AAs $(p=$ 0.2877) (Fig. 2-C). 
The relationship between WHO grade and OS was also analysed, and AAs showed poorer prognosis than DAs ( $p=0.007)$. For cases of "astrocytoma, grade 4" or "non-astrocytoma, grade 4", AA showed poorer survival curves compared with $D A$, and significant differences were identified with the Wilcoxon test $(p=$ 0.04 each), but not with the log-rank test $(p=0.09$ and $p=0.055$, respectively) (Fig. $3-A)$.

Log-rank testing showed significant correlations between OS and the following factors: age $>40$ years, EGFR gain/amplification, PDGFRA amplification, PTEN homozygous loss and loss, CDK4 gain/amplification, MDM2 homozygous loss and alteration, and TP53 homozygous loss (Table 2). These factors and TERTp mutation, EGFR amplification and the diagnosis of "astrocytoma, grade 4", as described above, were analysed by Cox proportional hazard modelling. After the stepwise procedure, three factors remained significant: PDGFRA amplification with the largest risk ratio (risk ratio 13.9, $\mathrm{p}=0.0022$ ), PTEN loss (risk ratio 9.75, $\mathrm{P}=0.0003$ ) with the most significance, and PTEN homozygous loss (risk ratio $6.97, \mathrm{p}=0.0329$ ) (Table 3). PDGFRA amplification was detected in 4 astrocytomas, and survival analysis showed a significant result $(p=0.043)$ (Fig. 3-B). PTEN loss was detected in 11 astrocytomas and 3 were homozygous losses and 8 were hemizygous losses, 6 of which were coincided with EGFR gains. A significant difference in OS was seen between astrocytomas with intact PTEN and those with PTEN loss $(p<0.0001)$, while there was no difference in OS between PTEN homozygous and PTEN hemizygous astrocytomas $(p=0.1255)$ (Fig. 3-C). No difference in survival of PTEN-loss astrocytomas was seen between presence or absence of EGFR gain $(\mathrm{p}=0.8133)$, and PTEN-loss astrocytomas showed poor prognosis compared with PTEN-intact astrocytomas irrespective of whether EGFR gain was present (with EGFR gain, $\mathrm{p}=0.0001$; without EGFR gain, $\mathrm{p}<0.0001$ ) (Fig. 3-D). 
Table 2

Results of Kaplan-Meier analysis and survival analysis about factors showing significance in survival analysis.

\begin{tabular}{|c|c|c|c|c|c|c|}
\hline & & \multirow[t]{2}{*}{$\mathbf{n}$} & \multirow[t]{2}{*}{ event } & \multirow{2}{*}{$\begin{array}{l}\begin{array}{l}\text { mos } \\
\text { (months) }\end{array} \\
(95 \% \mathrm{Cl})\end{array}$} & \multicolumn{2}{|l|}{$p$ value } \\
\hline & & & & & $\begin{array}{l}\text { log-rank } \\
\text { test }\end{array}$ & $\begin{array}{l}\text { Wilcoxon } \\
\text { test }\end{array}$ \\
\hline \multirow[t]{2}{*}{$\begin{array}{l}\text { CIMPACT-NOW } \\
\text { update } 3\end{array}$} & "grade 4" & 21 & 11 & $\begin{array}{l}29.0(16.9- \\
60.2)\end{array}$ & 0.0149 & 0.0428 \\
\hline & not "grade 4" & 19 & 6 & NR (24.5-NR) & & \\
\hline \multirow[t]{2}{*}{ TERTp } & mutant & 18 & 10 & $\begin{array}{l}30.4(15.8- \\
60.2)\end{array}$ & 0.0228 & 0.0384 \\
\hline & wild type & 22 & 7 & NR (24.5-NR) & & \\
\hline \multirow[t]{4}{*}{$E G F R$} & amplification & 8 & 4 & $\begin{array}{l}27.2(8.45- \\
29.0)\end{array}$ & 0.0401 & 0.1989 \\
\hline & no amplification & 32 & 13 & $\begin{array}{l}64.2(30.4- \\
N R)\end{array}$ & & \\
\hline & gain/amplification & 25 & 12 & $\begin{array}{l}29.0(21.3- \\
60.2)\end{array}$ & 0.0227 & 0.0466 \\
\hline & retained & 15 & 4 & NR (49.2-NR) & & \\
\hline \multirow[t]{2}{*}{ PDGFRA } & amplification & 4 & 3 & $\begin{array}{l}24.4 \text { (3.65- } \\
N R)\end{array}$ & 0.043 & 0.0793 \\
\hline & no amplification & 36 & 14 & $\begin{array}{l}60.2(29.0- \\
N R)\end{array}$ & & \\
\hline \multirow[t]{4}{*}{ PTEN } & homozygous loss & 3 & 3 & $\begin{array}{l}11.5(8.45- \\
15.9)\end{array}$ & $<0.0001$ & $<0.0001$ \\
\hline & $\begin{array}{l}\text { no homozygous } \\
\text { loss }\end{array}$ & 37 & 14 & $\begin{array}{l}60.2(29.0- \\
N R)\end{array}$ & & \\
\hline & lost & 11 & 9 & $\begin{array}{l}16.39 \text { (3.65- } \\
29.0)\end{array}$ & $<0.0001$ & $<0.0001$ \\
\hline & retained & 29 & 8 & NR (49.2-NR) & & \\
\hline \multirow[t]{2}{*}{ CDK4 } & gain/amplification & 10 & 7 & $\begin{array}{l}24.4(8.45- \\
29.0)\end{array}$ & 0.0006 & 0.0125 \\
\hline & retained & 30 & 10 & NR (49.2-NR) & & \\
\hline MDM2 & homozygous loss & 2 & 2 & $\begin{array}{l}16.4(8.45- \\
24.4)\end{array}$ & 0.008 & 0.022 \\
\hline
\end{tabular}

Abbreviations: $\mathrm{Cl}$, confidence interval; NR, not reached; mOS, median overall survival 


\begin{tabular}{|c|c|c|c|c|c|c|}
\hline & & \multirow[t]{2}{*}{$\mathbf{n}$} & \multirow[t]{2}{*}{ event } & \multirow{2}{*}{$\begin{array}{l}\text { moS } \\
\text { (months) } \\
(95 \% \mathrm{Cl})\end{array}$} & \multicolumn{2}{|l|}{$\mathrm{p}$ value } \\
\hline & & & & & $\begin{array}{l}\text { log-rank } \\
\text { test }\end{array}$ & $\begin{array}{l}\text { Wilcoxon } \\
\text { test }\end{array}$ \\
\hline & $\begin{array}{l}\text { no homozygous } \\
\text { loss }\end{array}$ & 38 & 15 & $\begin{array}{l}60.2(29.0- \\
N R)\end{array}$ & & \\
\hline & altered & 15 & 9 & $\begin{array}{l}24.5(11.5- \\
\text { NR) }\end{array}$ & 0.024 & 0.0279 \\
\hline & retained & 25 & 8 & NR (49.2-NR) & & \\
\hline \multirow[t]{2}{*}{ TP53 } & homozygous loss & 2 & 2 & $\begin{array}{l}16.4(8.45- \\
24.4)\end{array}$ & 0.008 & 0.022 \\
\hline & $\begin{array}{l}\text { no homozygous } \\
\text { loss }\end{array}$ & 38 & 15 & $\begin{array}{l}60.2(29.0- \\
N R)\end{array}$ & & \\
\hline \multirow[t]{2}{*}{ Age } & age $>40$ & 34 & 16 & $\begin{array}{l}49.2(24.4- \\
64.2)\end{array}$ & 0.0345 & 0.0524 \\
\hline & age $\leq 40$ & 6 & 1 & NR (30.4-NR) & & \\
\hline \multirow[t]{2}{*}{ WHO grade } & grade III & 22 & 13 & $\begin{array}{l}27.2(13.1- \\
49.2)\end{array}$ & 0.0071 & 0.004 \\
\hline & grade II & 18 & 4 & NR (55.9-NR) & & \\
\hline
\end{tabular}


Table 3

Results of Cox proportional hazard model analysis of OS for each factor.

\begin{tabular}{|lll|}
\hline & Risk ratio $(95 \% \mathrm{Cl})$ & p value \\
\hline WHO 2016, grade III & $6.46(0.942-44.4)$ & 0.0575 \\
\hline $0.218(0.00336-14.2)$ & 0.4744 & 0.3838 \\
\hline TERTp mutation & $5.14(0.129-204)$ & 0.7493 \\
\hline EGFR amplification & $0.709(0.0860-5.84)$ & 0.3749 \\
\hline EGFR gain/amplification & $3.04(0.261-35.4)$ & 0.6549 \\
\hline PDGFRA amplification & $2.17(0.0721-65.5)$ & 0.7730 \\
\hline PTEN homozygous loss & $1.65(0.0550-49.4)$ & 0.1283 \\
\hline PTEN loss & $5.93(0.598-58.8)$ & 0.9166 \\
\hline CDK4 gain/amplification & $1.16(0.0755-17.7)$ & 0.7467 \\
\hline MDM2 homozygous loss & $1.39(0.186-10.4)$ & 0.6978 \\
\hline MDM2 alteration & $1.47(0.212-10.2)$ & 0.1657 \\
\hline age over 40 years & $10.9(0.372-319)$ & \\
\hline *after stepwise procedure & & 0.0003 \\
\hline PTEN loss & $9.75(2.82-33.7)$ & 0.0329 \\
\hline PTEN homozygous loss & $6.97(1.17-41.5)$ & 0.0022 \\
\hline PDGFR amplification & $13.9(2.58-75.2)$ & \\
\hline Abbreviations: Cl, confidence interval & & \\
\hline The initial candidates (written above a bar) & are all factors for which Kaplan-Meier analysis or log-rank \\
\hline testing showed significant results. & & \\
\hline
\end{tabular}

\section{Discussion}

In the present study, the Sanger sequencing method could detect most of instances of "astrocytoma, grade 4", with the addition of MLPA successfully identifying all cases. Correlations between TERTp mutation and $7 p$ gain or $10 q$ loss have already been reported..$^{11}$ In the present study, the correlation analysis showed that TERTp mutation was associated with EGFR gain and PTEN loss, and + EGFR/PTEN astrocytoma always accompanied by TERTp mutation. This fact may result from a relationship between TERTp mutation, gain of chromosome 7, and loss of chromosome 10. The database of The Cancer Genome Atlas (TCGA) ${ }^{6}$ included 86 IDH-wild-type grade II or III gliomas, and TERTp status was examined in 56 cases. TERTp mutation was detected in 37 cases, and EGFR amplification was shown in 
15 out of 37 TERTp mutant gliomas and in 4 of 19 TERTp-wild-type gliomas. The status of $+7 /-10$ was evaluated in 55 of the 56 cases of TERTp mutant grade II or III glioma, with 27 cases showing $+7 /-10$. Among these 27 cases, 12 cases showed both TERTp mutation and EGFR amplification, 14 cases showed TERTp mutation alone, and the remaining 1 case showed EGFR amplification alone. In the present study, EGFR amplification was defined as different from EGFR gain, so + EGFR/-PTEN tumours never showed EGFR amplification. As in the present study, for IDH-wild-type grade II or III gliomas in TCGA database, TERTp status revealed almost all cases of "astrocytoma, grade 4" and the addition of EGFR status successfully identified all other cases of "astrocytoma, grade 4." Sanger sequencing and MLPA were thus thought to be reasonable methods for classifying IDH-wild-type lower-grade gliomas based on the recommendations from cIMPACT-NOW update $3 .^{15}$

WHO grade was a good marker of prognosis in the present study. IDH-wild-type AAs showed lower survival curve than IDH-wild-type DAs. Based on the WHO 2016 classification, glioma grade is partly affected by molecular factors including $1 \mathrm{p} / 19 \mathrm{q}$ codeletion and histone mutations, but gliomas are mainly classified according to histological characteristics, which are almost the same as in the WHO 2007 classification. Although our cohort showed no differences in sex, age, or other prognostic factors detected in multivariate analysis, treatment selections did differ between DAs and AAs. Patients with AAs tended to be initially treated with strong chemoradiation therapies like the Stupp regimen, ${ }^{23}$ and those with DAs tended to undergo observation alone more often; this might be one reason why the results of log-rank testing showing no statistical difference. The survival curve of DAs was definitely favourable compared with AAs in the early course, and the generalized Wilcoxon test showed a significant difference. Taking IDH-mutation status into consideration, WHO grade was reported as a significant factors for OS in lowergrade gliomas, ${ }^{11,24,25}$ and one of the studies showed that WHO grade had larger prognostic value in IDH wild-type astrocytomas compared with in $I D H$ mutant astrocytomas, with the authors proposing histological mitotic count as a significant predictor of prognosis. ${ }^{24}$ This fact supports that histological grading systems remain important, especially for $I D H$-wild-type astrocytoma.

The survival analysis showed TERTp mutation as a prognostic factor for OS in the group of all IDH-wildtype astrocytomas and IDH-wild-type DAs, and the diagnosis of "astrocytoma, grade 4" with EGFR amplification was significant only in all IDH-wild-type astrocytomas. TERTp mutation and EGFR amplification have been reported as characteristics of IDH-wild-type GBM and as unfavourable prognostic factors in IDH-wild-type astrocytomas in many studies, ${ }^{10-12,26}$ although a few studies have reported no significance. ${ }^{14,27}$ In our study, EGFR amplification was slightly more frequent in AAs than in DAs, while TERTp showed no difference between subtypes. TERTp mutation, EGFR amplification, and diagnosis of "astrocytoma, grade 4 " were significant factors in the group of all IDH-wild-type astrocytomas.

As mentioned above, WHO grade, TERTp mutation, EGFR amplification and diagnosis of "astrocytoma, grade 4" were good predictors of IDH-wild-type astrocytomas in Kaplan-Meier analysis, but Cox proportional hazard modelling detected no significance for OS in these factors. According to the Cox 
proportional hazard model of our cohort, copy number alteration of PTEN and PDGFRA amplification were significant predictors of OS.

Copy number alteration of PTEN was a strong predictor, as demonstrated by both the Kaplan-Meier method and Cox proportional hazard modelling. No different in OS was evident between PTEN hemizygous loss astrocytomas and PTEN homozygous loss astrocytomas, with both showing shorter OS than PTEN-intact astrocytomas. In addition, whether combined with EGFR gain or not, PTEN loss resulted in a significant difference in OS. PTEN loss is one of the typical genetic alteration of GBM, observed in about $30-40 \%{ }^{28,29}$ Some studies of prognostic factors in GBM patients have been published, but have been controversial about the significance of PTEN loss. ${ }^{27,30,31}$ However, in IDH-wild-type lower-grade astrocytomas, some papers have stated that PTEN loss is associated with poor prognosis, ${ }^{27,32}$ potentially because $P T E N$ is a tumour suppressor gene ${ }^{27,33}$ and inactivation of PTEN signalling is thus important to malignant progression to glioblastoma. ${ }^{34}$ The present study indicated PTEN loss as a strong predictor of poor prognosis in IDH-wild-type astrocytomas.

PDGFRA amplification showed a strong risk ratio in the present study, but only 4 AAs were included in the present study. PDGFRA amplification was also recognised as a characteristics of proneural GBM, which showed relatively good prognosis. ${ }^{29,35}$ The frequency of PDGFRA amplification in lower-grade glioma has only been reported from studies of small numbers of low-grade gliomas, ${ }^{36,37}$ and there was not enough evidence to conclude prognostic value of it. Strum et al. reported about subgrouping of GBMs based on the methylation profiles and compared them with other profiles of mutation and copy number status. ${ }^{35}$ PDGFRA amplification was more common in a methylation cluster, "RTK I", than the other four clusters. "RTK I" cluster also showed CDKN2A loss frequently. In the present study, a correlation between PDGFRA gain/amplification and CDKN2A homozygous loss was seen, and might imply that astrocytoma with alteration of PDGFR is associated with "RTK I" GBMs. In our cohort, no PDGFRA amplification was seen in DAs, but no difference in its frequency was evident between DAs and AAs because of the small number with PDGFRA amplification. Further studies are required to clarify the prognostic value of PDGFRA.

After the report of cIMPACT-NOW update 3, the genetic analyses such as copy number analysis have been extensively studied in lower grade gliomas, and it becomes clearer that several genetic markers are surely prognostic and that they need to be incorporated into clinical practice. In this context, our study has important implications by showing that such prognostic stratification can be done by direct sequencing and MLPA with a reasonable cost.

There are several limitations in the present study. First, the number of study population was small. Second, we did not perform external validation analysis. The result of MLPA analysis was not confirmed by other methodology such as CNV array.

\section{Conclusion}


The present study showed that the combination of Sanger sequencing and MLPA was sufficient to identify a subgroup of patients who has poorer prognosis in IDH-wild-type lower-grade astrocytoma. These patients were safely considered to have "astrocytoma, grade 4" according to the cIMPACT-NOW update 3 criteria. Our data also showed that PTEN loss and PDGFRA amplification are significant prognostic factors, and these genetic alterations would be good candidates for upcoming new classification. WHO grade is still useful to predict the clinical course of patients with IDH-wild-type gliomas.

\section{Methods}

\section{Ethics approval and consent to participate}

This study was carried out in accordance with the principles of the Declaration of Helsinki, and approval was obtained from the institutional review board at Kyoto University Hospital (approval number: G1124). Informed consent was obtained from all patients for inclusion in this study.

\section{Subjects}

The purpose of the present study was to assess the feasibility of the combination of Sanger sequencing and MLPA in classifying IDH-wild-type lower-grade astrocytomas, as diagnosed by the WHO 2016 classification, into a new classification recommended by cIMPACT-NOW update 3 , and to reveal prognostic factors for $I D H$-wild-type lower-grade astrocytoma.

The targets of the present study were $I D H$-wild-type astrocytomas surgically treated in Kyoto University Hospital. Inclusion criteria were as follows: 1) tumour samples after removal were stored as frozen or fresh specimens to maintain sufficient quality of DNA for extraction; 2 ) initial diagnosis was WHO grade II or grade III glioma; 3) Sanger sequence revealed no hot-point mutations in IDH1/2, H3F3A, or HIST1H3B; 4) MLPA showed no 1p/19q codeletion; and 5) informed consent was obtained.

\section{Sanger sequencing}

Tumour DNA was extracted from tumour specimens using NucleoSpin® Tissue (MACHEREY-NAGEL, Düren, Germany). Regions of interest for driver genes $3,16-18$ were amplified by PCR with gene-specific primers (Supplementary table S1) and TaKaRa Ex Taq® (TAKARA BIO, Shiga, Japan) (IDH1/2, H3F3A, and HIST1H3B) or AmpliTaq Gold 360 (Thermo Fisher Scientific, Waltham, MA) (TERTp) using an Applied Biosystems GeneAmp PCR System 9700 (Thermo Fisher Scientific). PCR products were processed by ExoSAP-IT (Thermo Fisher Scientific), then sequenced with sequencing primer (IDH1) or PCR forward primer as a sequencing primer (IDH2, H3F3A, HIST1H3B, TERTp) and a BigDye® Terminator V1.1 Cycle Sequencing Kit (Thermo Fisher Scientific) using the ABI 3130xL Genetic Analyzer (Thermo Fisher Scientific).

\section{MGMT promoter methylation analysis}


MGMTp methylation was assessed by quantitative methylation-specific PCR (qMSP), in accordance with previous reports. ${ }^{20,38}$ Genomic DNA samples were processed using an EZ DNA Methylation Gold Kit (Zymo Research Corporation, Irvine, CA). Methylated and unmethylated molecules were quantified by qMSP using a QuantStudio 12K Flex Real-Time PCR System (Thermo Fisher Scientific) with POWER SYBR ${ }^{\circledR}$ Green Master Mix (Thermo Fisher Scientific) and specific primers (Supplementary Table S1) ${ }^{39}$ according to the standard curve method. The methylation status of samples was determined from the ratio of methylated molecule using the cut-off value at $>1 \% .{ }^{20}$

\section{MLPA}

Copy number analyses of $1 \mathrm{p} / 19 \mathrm{q}, E G F R, P T E N, C D K N 2 A$ and PDGFRA were performed with MLPA according to the instructions from the manufacturer (SALSA MLPA KIT probemix P088-C2 for $1 p / 19 q$ analysis and SALSA MLPA KIT probemix P105-D2 for the others; MRC-Holland, Amsterdam, the Netherlands). ${ }^{19,20}$ MLPA with probemix P105-D2 can also analyse the alteration of MDM2, CDK4, NFKBIA, and TP53. Human genomic DNA G147A, G152A, and G304A (Promega, Madison, WI) were used as reference samples.

Data on MLPA were collected using an ABI 3130xL Genetic Analyzer (Thermo Fisher Scientific), then analysed using Coffalyzer.Net Software (MRC-Holland). Thresholds of copy number detection were chosen as reported previously ${ }^{19,40}$; thresholds of normal copy number were set at 0.8 to 1.2. A ratio of < 0.4 was set as the threshold for hemizygous and homozygous losses, and ratios $>2.0$ were defined as amplifications.

\section{Integrated diagnosis}

Tumour grading was performed histologically. Using all molecular pathological information, all cases received integrated diagnoses according to the 2016 WHO classification for central nervous system tumours. ${ }^{5}$

\section{Clinical outcomes}

Clinical data retrospectively collected from electronic records included age at diagnosis, sex, treatment protocol as chemotherapy or radiotherapy, and dates of surgery, last follow-up, and death.

\section{Classification of astrocytomas, IDH-wild type}

All IDH-wild-type astrocytomas were analysed by Sanger sequencing and MLPA to reveal the status of TERTp, EGFR gain or amplification, and PTEN loss. Classifications were performed in three steps. The first step was TERTp mutation, the second was EGFR amplification, and the third was + EGFR/-PTEN.

According to the recommendations of a previous study, ${ }^{15}$ "astrocytoma, grade 4 " was defined as IDHwild-type astrocytoma with any one or more of TERTp mutation, EGFR amplification, or + 7/-10. However, showing the whole chromosomal alteration with MLPA (with SALSA MLPA KIT probemix P105-D2) is 
impossible. Because EGFR and PTEN are located on chromosomes 7 and 10, respectively, tumours with $+7 /-10$ must have + EGFR/-PTEN. Therefore, in our classification system, $+E G F R /-P T E N$ was used as a criterion, and $+7 /-10$ was planned to be examined in + EGFR/-PTEN tumours.

\section{Statistical analysis}

All statistical analyses were performed using JMP Pro version 15.1.0 software (SAS Institute, Cary, NC). The difference of categorical variables was evaluated by Fisher's exact test or chi square test, and Student's t-test was used for continuous variables. For survival analyses, OS was defined as the interval between the initial operative day and the date of death, or last follow-up date on which the patient was known to be alive. Survival data were analysed using the Kaplan-Meyer curve and the $p$-value of survival in the present paper was determined by log-rank testing if there was no special comment, while generalized Wilcoxon test was used when appropriate. Multivariate analysis was performed by Cox proportional hazard modelling. The relationships between OS and following factors were analysed; age; MGMTp hyper-methylation; gain, amplification, and gain/amplification of EGFR, PDGFRA, and MDM2; hemizygous loss, homozygous loss and loss (including both of hemizygous and homozygous loss) of CDKN2A, PTEN, CDK4, MDM2, NFKBIA, and TP53. Differences were considered significant for values of $p$ $<0.05$.

\section{Abbreviations}

$\mathrm{AA}=$ anaplastic astrocytoma, grade 3

CDK4 = cyclin-dependent kinase 4

CDKN2A = cyclin-dependent kinase inhibitor 2A

$\mathrm{Cl}=$ confidence interval

CNV = copy number variation

CIMPACT-NOW update $3=$ the Consortium to Inform Molecular and Practical Approaches to CNS Tumour Taxonomy

$\mathrm{DA}=$ diffuse astrocytoma, grade 2

EGFR $=$ epidermal growth factor receptor

FFPE $=$ formalin-fixed paraffine-embedded

$\mathrm{GBM}=$ glioblastoma

$\mathrm{H} 3 \mathrm{~F} 3 \mathrm{~A}=\mathrm{H} 3$ histone, family $3 \mathrm{~A}$

HIST1H3B = histone cluster $1 \mathrm{H} 3$ family member $b$ 
$\mathrm{IDH}=$ isocitrate dehydrogenase

MDM2 = murine double minute 2

MGMTp = 06-methylguanine-DNA methyltransferase promoter

MLPA = multiplex ligation-dependent probe amplification

NFKBIA = nuclear factor kappa-B inhibitor alpha

OS = overall survival

PDGFRA = platelet-derived growth factor receptor $\mathrm{A}$

PTEN $=$ phosphatase and tensin homolog deleted from chromosome 10

qMSP = quantitative methylation-specific PCR

TCGA $=$ The Cancer Genome Atlas

TERT = telomerase reverse transcriptase

TERTp $=$ telomerase reverse transcriptase promoter

TP53 = tumour protein p53

\section{Declarations}

\section{Availability of data and material}

The datasets used and/or analysed during the current study available from the corresponding author on reasonable request.

\section{Acknowledgements}

This study included results based upon data generated by The Cancer Genome Atlas (TCGA) Research Network. We would like to acknowledge TCGA and their financial and material support in the development of the TCGA, as well as members of the consortium for their commitment to data sharing. Interpretations are the responsibility of the study authors.

\section{Authors' contributions}

Y.A. and Y.K. designed the study. Y.Ma., T.K, E.Y., T.S., and D.K. performed genetic 
analyses and data analyses. Y.T., M.T., Y.Mi., and S.M. collected samples. Y.Ma., Y.A. and Y.K.

wrote the manuscript. All authors read and approved the final manuscript.

\section{Competing interests}

Y.A. has received research funding research funding from Ono Pharmaceutical Co., Ltd.,Siemens, Philips, Sanofi, Nihon Medi-Physics, MitsubishiTanabe, Takeda, Stryker, Astellas Pharma, Taiho Pharma, and Pfizer outside the submitted work; honoraria from Nippon Kayaku, Behring, AbbVie, Novocure, UCB Japan, Ono Pharmaceutical, and Otsuka; and research funding and honoraria from Brainlab, Merck, Chugai, Eisai, Meiji Seika, Daiichi Sankyo, Zeiss, and CLS outside the submitted work. Y.Ma., E.Y., T.S., T.K., Y.T., M.T., D.K., Y.Mi., S.M., Y.K. declare no competing interests.

\section{Funding}

This work was supported by grants-in-aid from the Ministry of Education, Culture, Sports, Science, and Technology, Japan (project nos. 16K10754, 17K19724, 19K09505, and 19K22685).

\section{References}

1. Ostrom, Q. T. et al. CBTRUS Statistical Report: Primary Brain and Central Nervous System Tumors Diagnosed in the United States in 2007-2011. Neuro-Oncology16, iv1-iv63, doi:10.1093/neuonc/nou223 (2014).

2. Louis, D. N. et al. The 2007 WHO Classification of Tumours of the Central Nervous System. Acta Neuropathol.114, 97-109 https://doi.org/10.1007/s00401-007-0243-4 (2007).

3. Yan, H. et al. IDH1 and IDH2 mutations in gliomas. The New England journal of medicine.360, 765773 https://doi.org/10.1056/NEJMoa0808710 (2009).

4. Cairncross, J. G. et al. Specific Genetic Predictors of Chemotherapeutic Response and Survival in Patients With Anaplastic Oligodendrogliomas. JNCl: Journal of the National Cancer Institute.90, 1473-1479 https://doi.org/10.1093/jnci/90.19.1473 (1998).

5. Cavenee, W. K., Louis, D. N., Ohgaki, H. \& Wiestler, O. D. International Agency for Research on Cancer. WHO classification of tumours of the central nervous system. Revised 4th edition. edn(International Agency For Research On Cancer, 2016).

6. Ceccarelli, M. et al. Molecular Profiling Reveals Biologically Discrete Subsets and Pathways of Progression in Diffuse Glioma. Cell.164, 550-563 https://doi.org/10.1016/j.cell.2015.12.028 (2016). 
7. Eckel-Passow, J. E. et al. Glioma Groups Based on 1p/19q,IDH, andTERTPromoter Mutations in Tumors. New England Journal of Medicine.372, 2499-2508

https://doi.org/10.1056/nejmoa1407279 (2015).

8. Cancer Genome Atlas Research. Integrative Genomic Analysis of Diffuse Lower-Grade Gliomas. N Engl J Med.372, 2481-2498 https://doi.org/10.1056/NEJMoa1402121 (2015). N. et al. Comprehensive

9. Reuss, D. E. et al. Adult IDH wild type astrocytomas biologically and clinically resolve into other tumor entities. Acta Neuropathol.130, 407-417 https://doi.org/10.1007/s00401-015-1454-8 (2015).

10. Aibaidula, A. et al. Adult IDH wild-type lower-grade gliomas should be further stratified. Neurooncology.19, 1327-1337 https://doi.org/10.1093/neuonc/nox078 (2017).

11. Aoki, K. et al. Prognostic relevance of genetic alterations in diffuse lower-grade gliomas. NeuroOncology.20, 66-77 https://doi.org/10.1093/neuonc/nox132 (2018).

12. Wijnenga, M. M. J. et al. Molecular and clinical heterogeneity of adult diffuse low-grade IDH wild-type gliomas: assessment of TERT promoter mutation and chromosome 7 and 10 copy number status allows superior prognostic stratification. Acta Neuropathol.134, 957-959 https://doi.org/10.1007/s00401-017-1781-z (2017).

13. Stichel, D. et al. Distribution of EGFR amplification, combined chromosome 7 gain and chromosome 10 loss, and TERT promoter mutation in brain tumors and their potential for the reclassification of IDHwt astrocytoma to glioblastoma. Acta Neuropathol.136, 793-803 https://doi.org/10.1007/s00401-018-1905-0 (2018).

14. Tabouret, E. et al. Prognostic impact of the 2016 WHO classification of diffuse gliomas in the French POLA cohort. Acta neuropathologica.132, 625-634 https://doi.org/10.1007/s00401-016-1611-8 (2016).

15. Brat, D. J. et al. cIMPACT-NOW update 3: recommended diagnostic criteria for "Diffuse astrocytic glioma, IDH-wildtype, with molecular features of glioblastoma, WHO grade IV". Acta Neuropathol.136, 805-810 https://doi.org/10.1007/s00401-018-1913-0 (2018).

16. Arita, H. et al. Upregulating mutations in the TERT promoter commonly occur in adult malignant gliomas and are strongly associated with total 1p19q loss. Acta Neuropathol.126, 267-276 https://doi.org/10.1007/s00401-013-1141-6 (2013).

17. Arita, H. et al. A combination of TERT promoter mutation and MGMT methylation status predicts clinically relevant subgroups of newly diagnosed glioblastomas. Acta neuropathologica communications.4, 79 https://doi.org/10.1186/s40478-016-0351-2 (2016).

18. Bleeker, F. E. et al. IDH1 mutations at residue p.R132 (IDH1(R132)) occur frequently in high-grade gliomas but not in other solid tumors. Human mutation.30, 7-11 https://doi.org/10.1002/humu.20937 (2009).

19. Jeuken, J., Cornelissen, S., Boots-Sprenger, S., Gijsen, S. \& Wesseling, P. Multiplex Ligation-Dependent Probe Amplification. The Journal of Molecular Diagnostics.8, 433-443 https://doi.org/10.2353/jmoldx.2006.060012 (2006). 
20. Umehara, T. et al. Distribution differences in prognostic copy number alteration profiles in IDH-wildtype glioblastoma cause survival discrepancies across cohorts. Acta neuropathologica communications.7, 99 https://doi.org/10.1186/s40478-019-0749-8 (2019).

21. Weller, M. et al. Molecular classification of diffuse cerebral WHO grade II/III gliomas using genomeand transcriptome-wide profiling improves stratification of prognostically distinct patient groups. Acta Neuropathol.129, 679-693 https://doi.org/10.1007/s00401-015-1409-0 (2015).

22. Schouten, J. P. Relative quantification of 40 nucleic acid sequences by multiplex ligation-dependent probe amplification. Nucleic Acids Res.30, 57-57 https://doi.org/10.1093/nar/gnf056 (2002).

23. Stupp, R. et al. Radiotherapy plus concomitant and adjuvant temozolomide for glioblastoma. N Engl J Med.352, 987-996 https://doi.org/10.1056/NEJMoa043330 (2005).

24. Olar, A. et al. IDH mutation status and role of WHO grade and mitotic index in overall survival in grade II-III diffuse gliomas. Acta Neuropathol.129, 585-596 https://doi.org/10.1007/s00401-0151398-z (2015).

25. Petersen, J. K. et al. Targeted next-generation sequencing of adult gliomas for retrospective prognostic evaluation and up-front diagnostics. Neuropathology and applied neurobiology.https://doi.org/10.1111/nan.12645 (2020).

26. Akyerli, C. B. et al. Use of telomerase promoter mutations to mark specific molecular subsets with reciprocal clinical behavior in IDH mutant and IDH wild-type diffuse gliomas. Journal of Neurosurgery.128, 1102-1114 https://doi.org/10.3171/2016.11.jns16973 (2018).

27. Brito, C. et al. Clinical insights gained by refining the 2016 WHO classification of diffuse gliomas with: EGFR amplification, TERT mutations, PTEN deletion and MGMT methylation. BMC Cancer.19, https://doi.org/10.1186/s12885-019-6177-0 (2019).

28. Cancer Genome Atlas Research, N. Comprehensive genomic characterization defines human glioblastoma genes and core pathways. Nature.455, 1061-1068 https://doi.org/10.1038/nature07385 (2008).

29. Verhaak, R. G. W. et al. Integrated Genomic Analysis Identifies Clinically Relevant Subtypes of Glioblastoma Characterized by Abnormalities in PDGFRA, IDH1, EGFR, and NF1. Cancer Cell.17, 98110 https://doi.org/10.1016/j.ccr.2009.12.020 (2010).

30. Srividya, M. R. et al. Homozygous 10q23/PTEN deletion and its impact on outcome in glioblastoma: A prospective translational study on a uniformly treated cohort of adult patients. Neuropathology: official journal of the Japanese Society of Neuropathology.31, 376-383 https://doi.org/10.1111/j.1440-1789.2010.01178.x (2011).

31. Carico, C. et al. Loss of PTEN Is Not Associated with Poor Survival in Newly Diagnosed Glioblastoma Patients of the Temozolomide Era. PLOS ONE.7, e33684 https://doi.org/10.1371/journal.pone.0033684 (2012).

32. Sabha, N. et al. Analysis of IDH mutation, 1p/19q deletion, and PTEN loss delineates prognosis in clinical low-grade diffuse gliomas. Neuro-Oncology.16, 914-923 https://doi.org/10.1093/neuonc/not299 (2014). 
33. Cristofano, A. D., Pesce, B., Cordon-Cardo, C. \& Pandolfi, P. P. Pten is essential for embryonic development and tumour suppression. Nature Genetics.19, 348-355 https://doi.org/10.1038/1235 (1998).

34. Knobbe, C. B., Merlo, A. \& Reifenberger, G. Pten signaling in gliomas. Neuro-Oncology.4, 196-211 (2002).

35. Sturm, D. et al. Hotspot Mutations in H3F3A and IDH1 Define Distinct Epigenetic and Biological Subgroups of Glioblastoma. Cancer Cell.22, 425-437 https://doi.org/10.1016/j.ccr.2012.08.024 (2012).

36. Martinho, O. et al. Expression, mutation and copy number analysis of platelet-derived growth factor receptor A (PDGFRA) and its ligand PDGFA in gliomas. British Journal of Cancer.101, 973-982 https://doi.org/10.1038/sj.bjc.6605225 (2009).

37. Puputti, M. et al. Amplification of KIT, PDGFRA, VEGFR2, and EGFR in Gliomas. Mol. Cancer Res.4, 927-934 https://doi.org/10.1158/1541-7786.Mcr-06-0085 (2006).

38. Okita, Y. et al. (11)C-methinine uptake correlates with MGMT promoter methylation in nonenhancing gliomas. Clin Neurol Neurosurg.125, 212-216 https://doi.org/10.1016/j.clineuro.2014.08.004 (2014).

39. Esteller, M., Hamilton, S. R., Burger, P. C., Baylin, S. B. \& Herman, J. G. Inactivation of the DNA repair gene 06-methylguanine-DNA methyltransferase by promoter hypermethylation is a common event in primary human neoplasia. Cancer Res.59, 793-797 (1999).

40. Jeuken, J. et al. Robust detection of EGFR copy number changes and EGFR variant III: technical aspects and relevance for glioma diagnostics. Brain Pathol.19, 661-671 https://doi.org/10.1111/j.1750-3639.2009.00320.x (2009).

\section{Figures}


A)

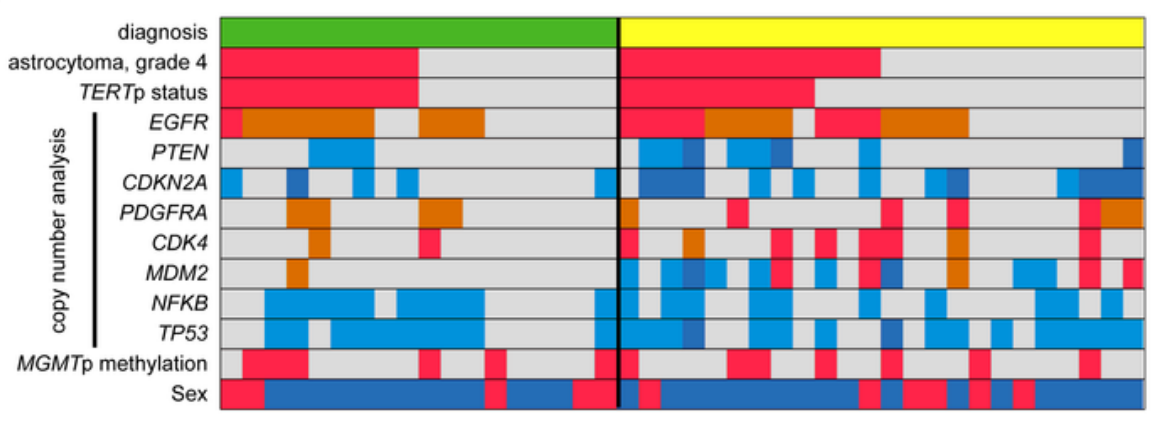

diagnosis

diagnosis of astrocytoma, grade 4

TERTp status

diffuse astrocytoma, IDH-wild type

not applicable

wild type anaplastic astrocytoma, $I D H$-wild type

copy number analysis

homozygous loss

hemizygous loss

MGMTp methylation status

intact

hyper methylated

Sex

intact

gain

amplification

B)

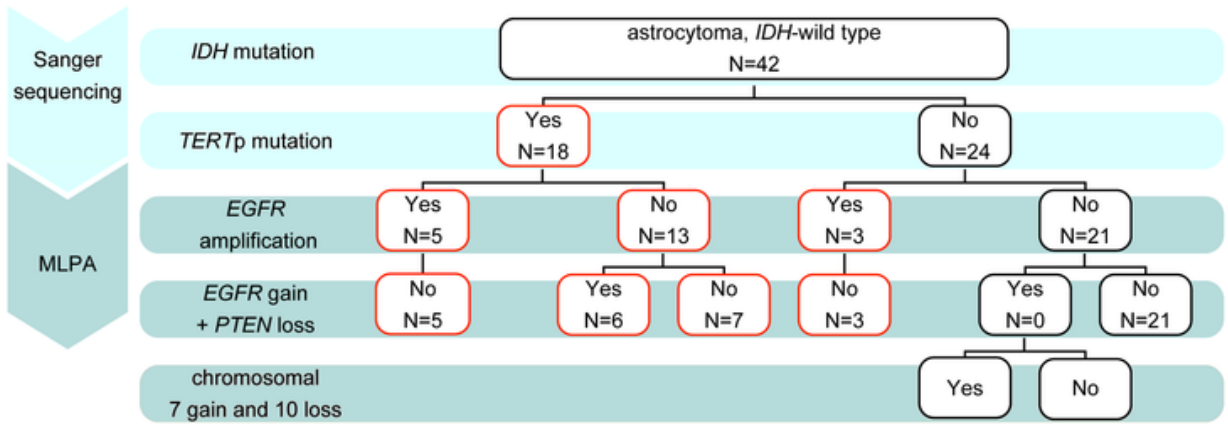

\section{Figure 1}

A) The cell plot shows characteristics of all 47 IDH-wild-type astrocytomas. B) The schema of three step classification of IDH-wild-type astrocytomas. TERTp mutation was examined by Sanger sequencing, and EGFR amplification and combination of EGFR gain and PTEN loss were examined by multiplex ligationdependent probe amplification (MLPA). The case with red square were diagnosed as "astrocytoma. grade $4 "$. 


\section{Figure 2}

A)
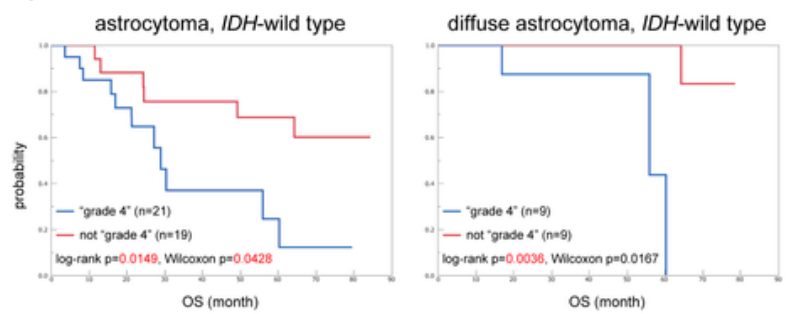

B)
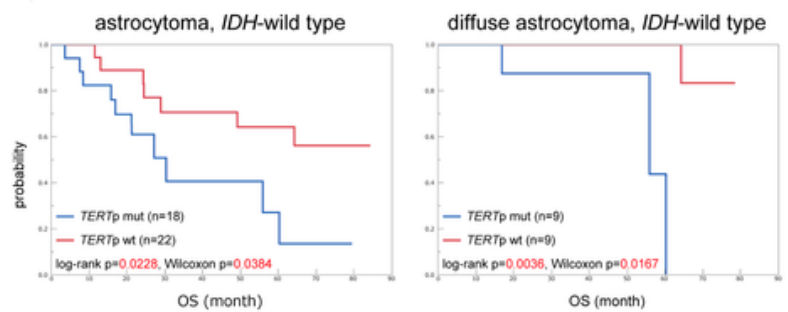

C)

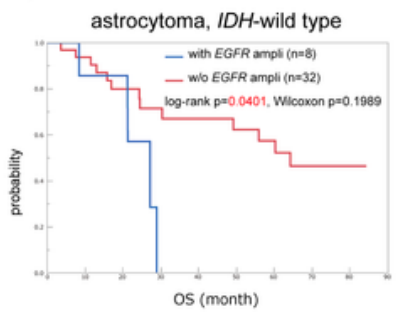

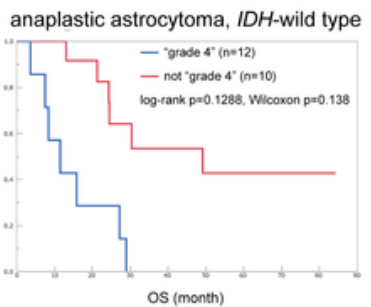

OS (month)

Makino et al.
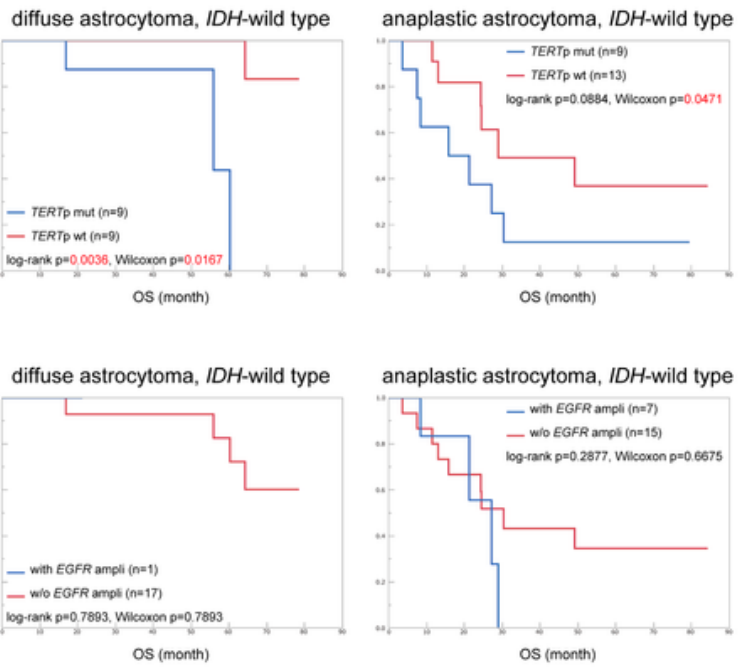

\section{Figure 2}

Survival analysis of 40 patients whose tumours were removed at their first surgery, were shown. The overall survival was analysed stratified by the following factors associated with the CIMPACT-NOW update 3 criteria; (A) the diagnosis of "astrocytoma, grade 4", (B) TERTp mutation, or (C) EGFR amplification, in the groups of all IDH-wild type astrocytomas, IDH-wild type diffuse astrocytomas, and 
IDH-wild type anaplastic astrocytomas. The $\mathrm{p}$ values were calculated log-rank test and Wilcoxon test, and $p<0.05$ was shown with red letters.

Figure 3

Makino et al.

A)
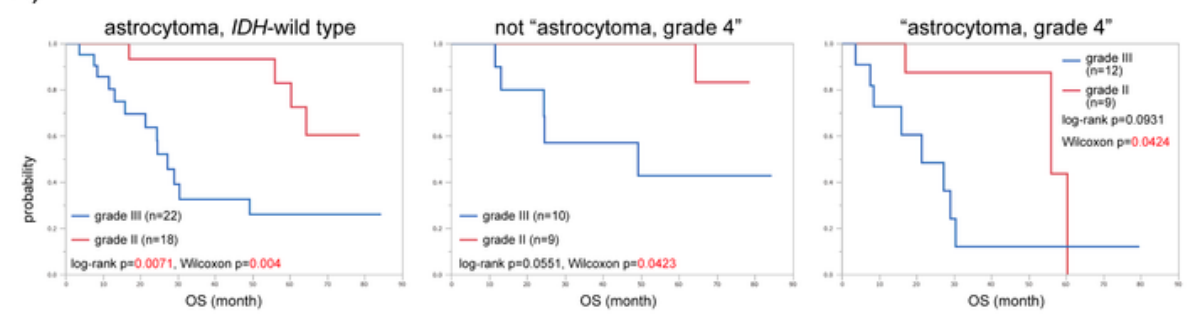

B)

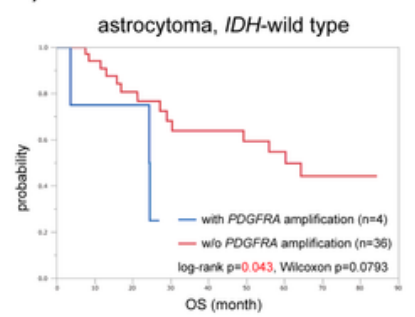

C)
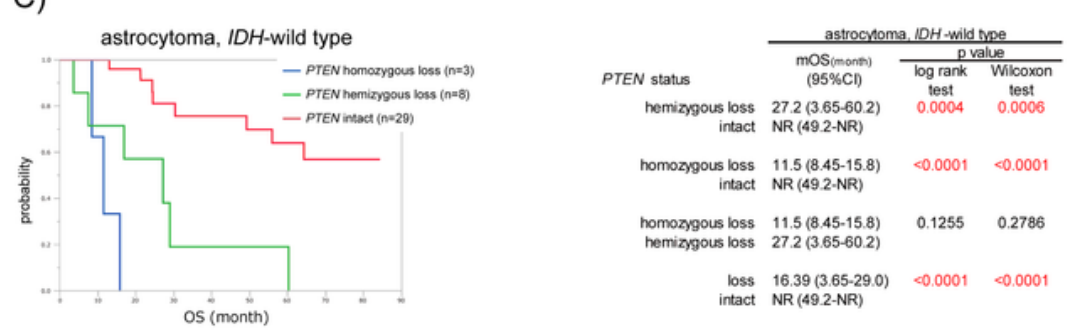

D)
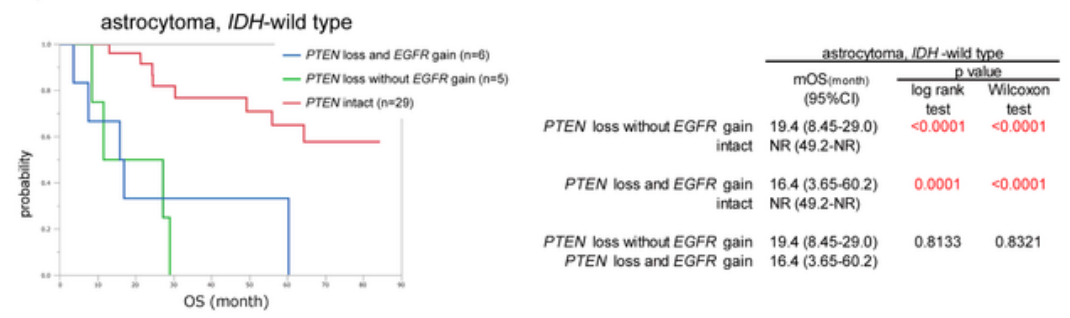

\section{Figure 3}

Survival analysis of 40 patients whose tumours were removed at their first surgery, were shown. (A) The overall survival of IDH-wild type diffuse astrocytomas (grade II) and anaplastic astrocytomas (grade III) were compared in the groups of all IDH-wild type astrocytomas, "astrocytoma, grade 4", and not- 
"astrocytoma, grade 4". The overall survival was also analysed stratified by the following factors associated with factors not included in cIMPACT-NOW update 3 criteria; (B) PDGFR amplification, (C) PTEN status (intact, hemizygous loss, or homozygous loss), and (D) the combination status of PTEN loss and EGFR gain. The $p$ values were calculated log-rank test and Wilcoxon test, and $p<0.05$ was shown with red letters.

\section{Supplementary Files}

This is a list of supplementary files associated with this preprint. Click to download.

- Supplementaltable120200204.docx 